\title{
Successful Treatment of Severe Aortic Stenosis With Transcatheter Aortic Valve Implantation in a Centenarian Patient
}

\author{
Yong-Joon Lee, MD ${ }^{1}$, Young-Ju Kim, MD ${ }^{1}$, Jung-Hee Lee ${ }^{1}$, Young-Guk Ko, MD ${ }^{1}$, \\ Sanghoon Shin, MD ${ }^{1}$, Geuru Hong, $\mathrm{MD}^{1}$, Sak Lee, $\mathrm{MD}^{2}$, Byung-Chul Chang, $\mathrm{MD}^{2}$, \\ Jae-Kwang Shim, $\mathrm{MD}^{3}$, Young-Ran Kwak, MD ${ }^{3}$, Myeong-Ki Hong, MD ${ }^{1}$ \\ Divisions of ${ }^{1}$ Cardiology, ${ }^{2}$ Thoracic and Cardiovascular Surgery, and ${ }^{3}$ Anesthesiology and Pain Medicine, \\ Severance Cardiovascular Hospital, Yonsei University Health System, Seoul, Korea
}

Transcatheter aortic valve implantation (TAVI) is a less invasive procedure to treat severe aortic valve stenosis than conventional surgical aortic valve replacement. Furthermore, TAVI has shown similar clinical outcomes as surgical treatment with less mortality and morbidities in elderly patients at high risk for conventional surgery. In this report, we describe case of successful TAVI using a CoreValve in a 103-year-old patient with symptomatic severe aortic valve stenosis.

Key Words: Aortic valve stenosis, Catheters, Heart valve prosthesis, Prosthesis implantation

\section{INTRODUCTION}

Aortic stenosis (AS) is the most common cause for obstruction of flow from the left ventricle into the aorta. It is present in $2 \%$ of those with age over 65 years and $4 \%$ of those with age over 85 years. Among various heartvalve diseases, AS is the most commonly acquired native valve disease afflicting the elderly population in western countries. As the Korean population is growing older, the prevalence of degenerative AS among the elderly in Korea is also increasing. Until recently, the standard treatment of severe AS has been surgical replacement of aortic valve. However, comorbidities and old age are associated with advanced procedure-related morbidity and mortality rates.

\footnotetext{
- Received: September 10, 2013 > Revised: December 23, 2013

- Accepted: December 30, 2013

Address for correspondence: Myeong-Ki Hong, MD, PhD

Division of Cardiology, Severance Cardiovascular Hospital,

Severance Biomedical Science Institute, Yonsei University College of Medicine, 50 Yonsei-ro, Seodaemun-gu, Seoul 120-752, Korea

Tel: +82-2-2228-8460, Fax: +82-2-2227-7732

E-mail: mkhong61@yuhs.ac
}

About one third of patients with severe AS and older than 75 years are not referred to a surgeon because of the high surgical risk ${ }^{1)}$.

The development of a catheter-based therapy for heart valve disease is rapidly evolving, especially in AS. Transcatheter aortic valve implantation (TAVI) allows the replacement of aortic valve without asternotomy or cardiopulmonary support. In this report, we describe a 103-yearold Korean patient treated by TAVI using a CoreValve (Medtronic, Minneapolis, MN, USA).

\section{CASE REPORT}

A 103-year-old male presented with dyspnea, cough and pinkish sputum. The patient had a history of hypertension and coronary artery disease with previous stent insertion at right coronary artery 5 years ago. He had been diagnosed with severe AS and came to our hospital for consideration of TAVI. On admission his blood pressure and pulse rate were $145 / 51 \mathrm{mmHg}$ and $98 / \mathrm{min}$. His body weight and height was $42.1 \mathrm{~kg}$ and $150 \mathrm{~cm}$, respectively. The 12-lead electrocardiogram (ECG) on admission 
showed sinus rhythm with right bundle branch block and findings suggestive of left ventricular hypertrophy with strain on lateral leads. Cardiomegaly and nodular opacity in right apex and hilum were noted on the chest x-ray. Transthoracic echocardiography revealed severe degenerative change of aortic valve with severe calcification (Fig. 1A, C). The aortic valve area was $0.36 \mathrm{~cm}^{2}$ with a mean systolic pressure gradient of $65 \mathrm{mmHg}$ and peak systolic pressure gradient of $109 \mathrm{mmHg}$ compatible with severe AS. The left ventricular end diastolic and systolic diameter were 48 and $38 \mathrm{~mm}$ with slightly reduced systolic function (ejection fraction 47\%). There was mild pulmonary hypertension with an estimated right ventricular systolic pressure of $37 \mathrm{mmHg}$. Computed tomography (CT) images demonstrated heavily calcified aortic valve with annulus diameter ranging from 22 to $30 \mathrm{~mm}$ and annulus perimeter of $80.6 \mathrm{~mm}$. The CT angiography demonstrated iliac arteries with minimum diameter of greater than 6.5 $\mathrm{mm}$ and common femoral arteries with minimum diameter of greater than $7.5 \mathrm{~mm}$ on both sides. There was no significant stenosis or calcification in iliac and common femoral arteries. Coronary angiography showed 90\% instent restenosis of previously implanted stent at right coronary artery ostium. A pulmonary function test revealed no obstructive airway disease. A multidisciplinary heart team consisted of interventional cardiologists, cardiac surgeons, anesthesiologists and imaging specialists evaluated the case and approved TAVI in this patient.

The procedure was carried out in a hybrid operating room under general anesthesia. Before TAVI procedure, in-stent restenosis of the proximal right coronary artery was first treated successfully with balloon angioplasty via the right common femoral artery (CFA). The vascular access for the 18-Fr CoreValve delivery catheter was obtained at the left CFA with standard percutaneous access techniques. A 6-Fr introducer sheath was inserted through the left CFA after preparing percutaneous closure using two preloaded suture devices (Perclose Proglide, Abbott Laboratories, Abbott Park, IL, USA). A pigtail catheter was inserted through the right CFA and positioned at the aortic root for the aortography during the procedure. A 0.032 inch wire was passed through the aortic valve into the left ventricle using an AL1 catheter. The AL1 catheter was exchanged to a pigtail catheter. A 0.035 inch preshaped superstiff wire was inserted in the left ventricle through pigtail catheter. The 6-Fr introducer sheath was exchan-
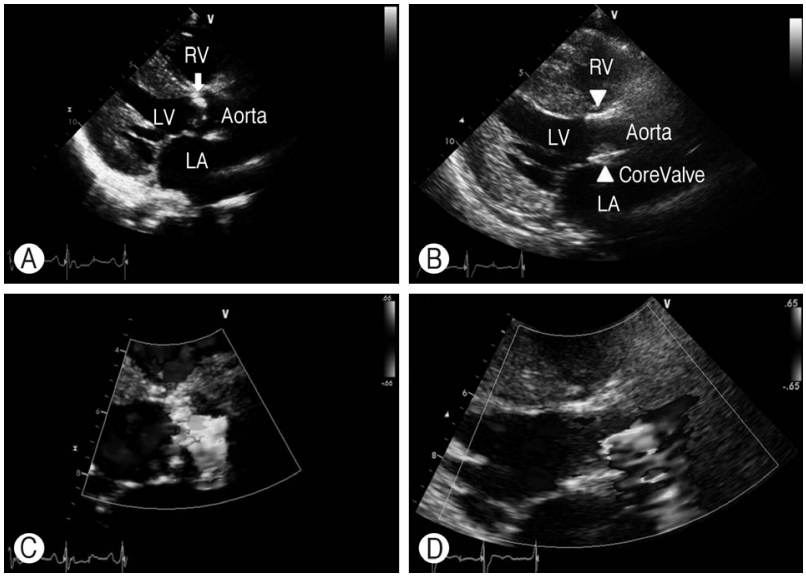

Fig. 1. (A) Transthoracic echocardiogram with a parasternal long axis view of the aortic valve. Sclerocalcifiedstenotic aortic valve before TAVI (arrow). (B) Implanted CoreValve after TAVI (arrowheads). (C) Doppler echocardiogram showing severe aortic stenosis with turbulence across the valve before TAVI (peak systolic pressuregradient of $109 \mathrm{mmHg}$ ). (D) After TAVI (peak systolic pressuregradient of $17 \mathrm{mmHg}$ ). LA, left atrium; LV, left ventricle; RV, right ventricle; TAVI, transcatheter aortic valve implantation.

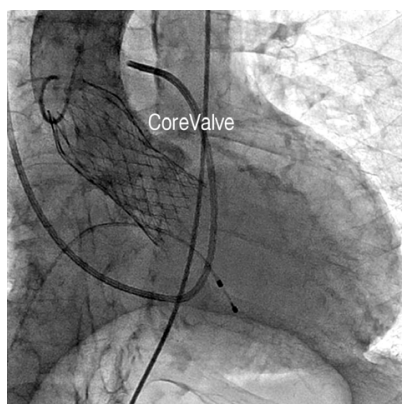

Fig. 2. Fluoroscopic image of the CoreValve positioned within the aortic valve.

ged to a 18-Fr sheath. A 29-mm CoreValve was loaded in a delivery catheter and the delivery catheter was inserted through the 18-Fr sheath into the aortic valve. Under angiographic guidance, the CoreValve was positioned within the aortic valve and slowly deployed (Fig. 2). After CoreValve deployment, transesophageal echocardiography (TEE) showed moderate degree of paravalvular leak. Therefore, postdilation was performed using a $25 \times 40$ $\mathrm{mm}$ balloon (Maxi, Cordis, Diegem, Belgium) during rapid right ventricular pacing. Final postprocedural TEE demonstrated a mild-to-moderate degree of paravalvular leak. The vascular access site for TAVI was closed by tightening the Perclose knots. The procedure was completed without complications. 
After the procedure, the patient was observed in an intensive careunit with continuous ECG monitoring and temporary pacemaker back-up. The follow-up transthoracic echocardiography showed the well functioning bioprosthetic aortic valve (Fig. 1B, D) with reduced peak and mean pressure gradient over the aortic valve 17 and $9 \mathrm{mmHg}$, respectively. No significant conduction abnormalities were observed except for intermittent complete atrio-ventricular block. The patient was discharged from the hospital at day 8 post procedure.

\section{DISCUSSION}

This is the case of the oldest patient in Korea who underwent TAVI procedure so far. The current indications for TAVI are as follows: (1) severe AS (aortic valve area: $<1 \mathrm{~cm}^{2}$, mean pressure gradient $>40 \mathrm{mmHg}$, severe symptoms); (2) contraindication for surgical valve replacement. The contraindications for TAVI areas follows: (1) mild to moderate AS; (2) asymptomatic patients; (3) life expectancy <1 year; (4) surgical aortic valve replacement possible, but patient refused; (5) aortic annulus $<18$ or $>25 \mathrm{~mm}$ (ballon-expandable) and $<20$ or $>27 \mathrm{~mm}$ (selfexpandable); (6) bicuspid aortic valve; (7) asymmetric heavy valvular calcification; (8) Aortic root $>45 \mathrm{~mm}$ at the aortotubular junction; (9) presence of left ventricular apical thrombus ${ }^{2}$. In addition, patient's general condition and comorbities should also be taken into consideration. It is common that very old patients have multiple comorbities over all kinds of organs. Although our patient was over 100 years old and had symptomatic severe AS, he was able to ambulate without assistance and had no mental problems such as dementia. Therefore, our multidisciplinary cardiovascular team decided to proceed with TAVI instead of surgery or medical treatment.

Treatment with TAVI is generally associated with lower rates of death from any cause, as compared with standard therapy. The PARTNER (Placement of AoRTictraNscathetER valves) trial ${ }^{3,4)}$ has evaluated the transcatheter aortic valve replacement in patients with AS who are considered either high risk (Cohort A, 699 patients) or inoperable (Cohort B, 358 patients) for conventional openheart surgery. Thirty-day mortality for PARTNER A was $3.4 \%$ for transcatheter aortic valve replacement and 6.5\% for aortic valve replacement; 1 -year mortality was $24.2 \%$ and $26.8 \%(\mathrm{p}=0.001)$, respectively. For PARTNER B, thirty-day mortality was $5.0 \%$ for transcatheter aortic valve replacement and 2.8\% for medical treatment; 1-year mortality was $30.7 \%$ vs. $50.7 \%$ ( $p<0.001)$, respectively. In addition to decrease in mortality and morbidity, TAVI procedures improved clinical symptoms and physical performance. Grimaldi et al. ${ }^{5)}$ assessed the improvement of quality of life (QoL) in 145 octogenarians who underwent TAVI for AS or isolated AR instead of surgery since their comorbities were unacceptable for surgery. QoL was assessed by questionnaires concerning physical and psychic performance. As a result, $49 \%$ walked an-assisted, $79 \%$ reported self-awareness improvement; QoL was reported as "good" in $58 \%$, "acceptable according to age" in 34\% and "bad" in $8 \%$.

Currently, self-expanding CoreValve and balloon-expanding SAPIEN valve are widely used for TAVI procedure. Although there has been no randomized comparison study between these two bioprosthetic valves, technical success rates and major clinical outcomes appeared to be similar except for conduction disorders such as left bundle branch or atrio-ventricular block and pacemaker implantation (20-30\% versus 3-5\%) which are more frequently observed in patients treated with the CoreValve versus SAPIEN valve $^{6-10)}$. The two valves have totally different deployment methods. Whereas SAPIEN valve is deployed with instant balloon inflation, CoreValve is inserted by slow release for self-expansion with more room for adjustment. The choice of the valve is currently more dependent on the operator's technical preference. In our case, the patient was discharged from the hospital at day 8 post TAVI without significant complication except for the transient atrio-ventricular block. His dyspnea symptom has been significantly improved after the procedure.

In summary, this is the case of the oldest patient in Korea with severe AS who has been successfully treated by TAVI. In very elderly patients at high risk for conventional surgery, TAVI appears to be an effective and safe alternative treatment modality ${ }^{3,4,11,12)}$.

\section{REFERENCES}

1. Goncalves A, Marcos-Alberca P, Almeria C, Feltes G, Hernandez-Antolin RA, Rodriguez E, et al. Quality of life improve- 
ment at midterm follow-up after transcatheter aortic valve implantation. Int J Cardiol 2013;162:117-22.

2. Akin I, Kische S, Schneider H, Rehders TC, Nienaber CA, Ince $\mathrm{H}$. Current indications for transcatheter aortic valve implantation. In: Chen YF, editor. Aortic valve. Rijeka: InTech; 2011. p.195-210.

3. Smith CR, Leon MB, Mack MJ, Miller DC, Moses JW, Svensson LG, et al. Transcatheter versus surgical aortic-valve replacement in high-risk patients. N Engl J Med 2011;364: 2187-98.

4. Leon MB, Smith CR, Mack M, Miller DC, Moses JW, Svensson LG, et al. Transcatheter aortic-valve implantation for aortic stenosis in patients who cannot undergo surgery. N Engl J Med 2010;363:1597-607.

5. Grimaldi A, Figini F, Maisano F, Montorfano M, Chieffo A, Latib A, et al. Clinical outcome and quality of life in octogenarians following transcatheter aortic valve implantation (TAVI) for symptomatic aortic stenosis. Int J Cardiol 2013;168:281-6.

6. Laynez A, Ben-Dor I, Barbash IM, Hauville C, Sardi G, Maluenda G, et al. Frequency of conduction disturbances after Edwards SAPIEN percutaneous valve implantation. Am J Cardiol 2012;110:1164-8.

7. Roten L, Stortecky S, Scarcia F, Kadner A, Tanner H, Delacretaz E, et al. Atrioventricular conduction after trans- catheter aortic valve implantation and surgical aortic valve replacement. J Cardiovasc Electrophysiol 2012;23:1115-22.

8. Nuis RJ, Van Mieghem NM, Schultz CJ, Tzikas A, Van der Boon RM, Maugenest AM, et al. Timing and potential mechanisms of new conduction abnormalities during the implantation of the Medtronic CoreValve System in patients with aortic stenosis. Eur Heart J 2011;32:2067-74.

9. Spargias K, Toutouzas K, Chrissoheris M, Synetos A, Halapas A, Paizis I, et al. The Athens TAVR Registry of newer generation transfemoral aortic valves: 30 -day outcomes. Hellenic J Cardiol 2013;54:18-24.

10. Carnicero A, Ben-Dor I, Hauville C, Maluenda G, Barbash I, Kitabata $\mathrm{H}$, et al. Incidence of conduction disturbances and need for permanent pacemaker after transcatheter implantation of an Edwards Sapien aortic valve prosthesis. J Am Coll Cardiol 2012;59(13 Suppl):E1983.

11. Sarkar K, Ussia GP, Tamburino C. Transcatheter aortic valve implantation for severe aortic regurgitation in a stentlessbioprosthetic valve with the Core Valve revalving system-technical tips and role of the Accutrak system. Catheter Cardiovasc Interv 2011;78:485-90.

12. Jo HC, Jung SM, Jang JW. A case of severe aortic stenosis patient with high operative risk treated by transcatheter aortic-valve implantation. J Korean Geriatr Soc 2013;17:90-4. 